\title{
Precisely timed signal transmission in neocortical networks with reliable intermediate-range projections
}

\author{
Martin Paul Nawrot ${ }^{1,2,3}$, Philipp Schnepel ${ }^{4}$, Ad Aertsen $^{3,4}$ and Clemens Boucsein ${ }^{3,4}$ * \\ Neuroinformatics and Theoretical Neuroscience, Institute of Biology-Neurobiology, Freie Universität Berlin, Berlin, Germany \\ 2 Bernstein Center for Computational Neuroscience Berlin, Berlin, Germany \\ ${ }^{3}$ Bernstein Center for Computational Neuroscience Freiburg, Albert-Ludwigs-University, Freiburg, Germany \\ ${ }^{4}$ Neurobiology and Biophysics, Institute of Biology III, Albert-Ludwigs-University, Freiburg, Germany
}

Edited by:

Michael Brecht, Humboldt University Berlin, Germany

\section{Reviewed by:}

Fritjof Helmchen, University of Zurich, Switzerland

Carl Petersen, Ecole Polytechnique

Fédérale de Lausanne, Switzerland

\section{*Correspondence:}

Clemens Boucsein, Neurobiology and Biophysics, Institute of Biology III,

Albert-Ludwigs-University,

Schänzlestrasse 1, D-79104 Freiburg,

Germany.

e-mail:clemens.boucsein@biologie. uni-freiburg.de
The mammalian neocortex has a remarkable ability to precisely reproduce behavioral sequences or to reliably retrieve stored information. In contrast, spiking activity in behaving animals shows a considerable trial-to-trial variability and temporal irregularity. The signal propagation and processing underlying these conflicting observations is based on fundamental neurophysiological processes like synaptic transmission, signal integration within single cells, and spike formation. Each of these steps in the neuronal signaling chain has been studied separately to a great extend, but it has been difficult to judge how they interact and sum up in active sub-networks of neocortical cells. In the present study, we experimentally assessed the precision and reliability of small neocortical networks consisting of trans-columnar, intermediate-range projections (200-1000 $\mu \mathrm{m})$ on a millisecond time-scale. Employing photo-uncaging of glutamate in acute slices, we activated a number of distant presynaptic cells in a spatio-temporally precisely controlled manner, while monitoring the resulting membrane potential fluctuations of a postsynaptic cell. We found that signal integration in this part of the network is highly reliable and temporally precise. As numerical simulations showed, the residual membrane potential variability can be attributed to amplitude variability in synaptic transmission and may significantly contribute to trial-to-trial output variability of a rate signal. However, it does not impair the temporal accuracy of signal integration. We conclude that signals from intermediate-range projections onto neocortical neurons are propagated and integrated in a highly reliable and precise manner, and may serve as a substrate for temporally precise signal transmission in neocortical networks.

Keywords: cell assembly, coincidence detector, cortex, dynamic photo stimulation, synaptic variability, temporal coding, rate coding

\section{INTRODUCTION}

The reliability and temporal precision of signal propagation between neurons is a major constraint for different coding strategies in neuronal networks. In systems that rely on rate coding, input-output functions of neurons are classically described as ratios of mean firing rates, and the precise timing of individual action potentials is not considered a meaningful parameter (Shadlen and Newsome, 1994, 1998). In these systems, synchrony of presynaptic action potentials and reliable synaptic transmission have even been implicated to deteriorate the information content of the postsynaptic spike train (Zador, 1998). For the functioning of a temporal code in neuronal networks, on the other hand, the precision and reliability of synaptic integration is a prerequisite (Abeles, 1991; Konig et al., 1996; Mainen and Sejnowski, 1995; Nowak et al., 1997; Roy and Alloway, 2001), and without exact spike timing in the millisecond range, synchronous activity among neurons that putatively form a functional cell assembly (Braitenberg, 1978; Harris, 2005; von der Malsburg, 1986), is likely to dilute and, eventually, vanish in networks designed to work with spike timing (Abeles, 1991; Diesmann et al., 1999; Mehring et al., 2003). The coding strategy utilized in neocortical networks might, thus, be reflected in its ability or inability to reliably propagate signals either as spike rates or precisely timed spikes.
At the cellular level, the neuronal signal propagation chain can be divided into four functional components: synaptic transmission, dendritic integration of synaptic events, the spike generating mechanism, and the axonal propagation of action potentials. Each of these components has its own physiological characteristics, and the integral of all four determines the overall behavior of an individual neuron. The spike generating mechanism in cortical cells has been shown to react reliable and with high temporal precision to fast transient membrane potential fluctuations in a series of in vitro studies that employed noise current injection (Berger and Luscher, 2003; Boucsein et al., 2009; Köndgen et al., 2008; Mainen and Sejnowski, 1995; Nowak et al., 1997; Silberberg et al., 2004). Action potential propagation itself seems not to influence precision in the neocortex substantially. Even though there have been reports on failures of action potential propagation at axonal branching points in cultured dorsal root ganglion cells (Lüscher et al., 1994) and in the hippocampus (Debanne et al., 1996), for neocortical neurons it has been shown that action potentials reliably invade all axonal branches (Cox et al., 2000). Studies concerning synaptic physiology have uncovered results specific to the particular projections that were considered. Synaptic reliability (i.e. the reverse of the failure rate) and amplitude variability in local 
connections (up to $200 \mu \mathrm{m}$ lateral distance between cell bodies) seems to depend on pre- and postsynaptic cell types and on layer. Reliability of projections from layer $2 / 3$ pyramidal cells range from only $30 \%$ when contacting bitufted cells, over $78 \%$ for pyramidto pyramid projections, to $96 \%$ for projections onto multipolar cells (Koester and Johnston, 2005); Projections from layer 4 stellate cells onto layer $2 / 3$ pyramids (Silver et al., 2003) and between layer 4 interneurons (Feldmeyer et al., 1999) showed a reliability of $95 \%$, and projections from layer 5 pyramids onto layer 4 cells or between layer 5 pyramids around 90\% (Frick et al., 2008; Gil et al., 1999; Markram et al., 1997), with a tendency towards more reliable connections with increasing strength. Those quantifications of synaptic physiology have been performed using paired recordings of closely neighboring cells in acute brain slices, and their heterogeneity makes it difficult to estimate the overall reliability of signal integration in local synaptic circuits. The reliability and amplitude variability of intermediate-range, trans-columnar projections have, on the other hand, not been investigated due to methodological constraints, i.e. the difficulty to find and record from coupled cells at distances of several hundred microns. In layer $2 / 3$, connection probability was predicted to drop below $15 \%$ or might even approach values close to zero at distances over $200 \mu \mathrm{m}$, depending on the location of the connected cells (Hellwig, 2000; Song et al., 2005; Thomson and Bannister, 2003). However, the absolute number of projections from cells at intermediate distances might still be substantial. When considering the close to cubic increase of the number of potential presynaptic cells with increasing distance, even a low connection probability can lead to an increasing number of presynaptic cells with distance (Holmgren et al., 2003; Matsuzaki et al., 2008). Intermediate-range projections may, thus, play an important role for information processing on a scale exceeding the local axonal and dendritic arborizations of pyramidal neurons, often referred to as a 'column', because they may link different stimulus features in primary sensory cortices across columns (Buzas et al., 2006; Mitchison and Crick, 1982; Staiger et al., 2004; Weliky and Katz, 1994).

In contrast to synaptic physiology and spike generation, there are very few studies that experimentally addressed the reliability of dendritic integration of multiple synaptic inputs. This is mainly due to experimental limitations with respect to the controlled generation of postsynaptic events at multiple dendritic sites. Conventional approaches using glutamate application through glass pipettes or photolysis of an excitatory neurotransmitter (photostimulation) using thin optical fibers typically allow to target only few (typically two or three) independent dendritic sites. New methods for patterned photostimulation provide an alternative experimental approach. For example, Shoham et al. (2005) presented a technique that achieves spatially and temporally patterned photostimulation at various sites within the dendritic tree of a postsynaptic cell and demonstrated its application to the statistical analysis of dendritic integration in a hippocampal CA1 pyramidal neuron, where mostly linear integration was observed. However, if strong activity impinges on the same basal dendrite, nonlinear events like NMDA-spikes have been observed with the application of a similar technique (Losonczy and Magee, 2006). Ca-spikes in the apical dendrite of pyramidal cells have been described in great detail (Magee, 2000; Williams and Stuart, 2003) but these nonlinear mechanisms come into play only at higher input rates than achievable with our experimental paradigm and were, thus, not taken into account in the present study. We experimentally assess the question how reliable and precise a neocortical neuron can integrate synaptic activity that stems from the dynamic activation of multiple trans-columnar projections in an intact neocortical sub-network using our recently developed technique of dynamic photo stimulation (DPS; Boucsein et al., 2005). Similar to early instances of static glutamate uncaging techniques (Callaway and Katz, 1993), our technique relies on the spatially controlled activation of presynaptic cells in the acute brain slices. The novelty of DPS lies in its ability to sequentially activate distant presynaptic cells in a sufficiently fast manner to impose a barrage of synaptic inputs that converge onto the postsynaptic cell. Our experimental paradigm employs functional projections with their natural occurring projection pattern, i.e. the number of functional synaptic contacts per cell-to cell connection, as well as the natural synaptic physiology (synaptic weights, amplitude variability, failure rate, etc.). We first characterized the synaptic strength and variability of individual intermediate-range intracortical projections in a conventional photostimulation approach. Second, we employed DPS to asses the reproducibility of membrane potential fluctuations in postsynaptic cells in response to repeated stimulation of those projections with a frozen spatio-temporal activation pattern of a set of presynaptic cells. We found unexpectedly precise and reliable synapses which mediated a temporally precise and reliable response. These transmission properties may possibly favor the use of a precise temporal coding scheme in trans-columnar neocortical networks.

\section{MATERIALS AND METHODS PREPARATION OF BRAIN SLICES}

For electrophysiological recordings, Long Evans rats (P17-25) were anesthetized with Isoflurane and decapitated. Brains were transferred to ice-cold preparation buffer and slices of $350 \mu \mathrm{m}$ thickness were cut with a vibratome (Dosaka, Kyoto, Japan) and stored at $33^{\circ} \mathrm{C}$ for $1 \mathrm{~h}$. Slices were transferred to the recording chamber and constantly superfused with artificial cerebro-spinal fluid (ACSF) containing (in $\mathrm{mM}$ ): $125 \mathrm{NaCl}, 2.5 \mathrm{KCl}, 1 \mathrm{MgCl}_{2}$, $2 \mathrm{CaCl}_{2}, 25 \mathrm{NaHCO}_{3}, 1.25 \mathrm{NaH}_{2} \mathrm{PO}_{4}$ and 25 glucose, $\mathrm{pH} 7.4$, gassed with carbogen $\left(95 \% \mathrm{O}_{2}\right.$ and $\left.5 \% \mathrm{CO}_{2}\right)$, at a flow rate of $4-6 \mathrm{ml} / \mathrm{min}$. Temperature was adjusted to $32-34^{\circ} \mathrm{C}$, and during uncaging experiments, the ACSF containing $400 \mu \mathrm{M} \gamma$-CNB-caged L-glutamic acid (G-7055, Molecular Probes, Leiden, the Netherlands) was re-circulated. To prevent the activation of metabotropic glutamate receptors, which can otherwise trigger apoptotic events, (RS)-MCPG (Tocris, Köln, Germany) at a concentration of $500 \mu \mathrm{M}$ was added to the ACSF. All chemicals were purchased from Sigma (Deisenhofen, Germany) if not otherwise noted. Animal treatment was according to the Freiburg University's and German guidelines on the use of animals in research.

\section{ELECTROPHYSIOLOGICAL RECORDINGS AND STAINING PROCEDURES}

Whole-cell patch-clamp recordings from layer 5 pyramidal cells in coronal slices of the somato-sensory cortex were established using standard procedures. Special care was taken to avoid recordings from cells close to the slice surface, which can have dendrites cut close to the soma. Recording depth was $146 \pm 65 \mu \mathrm{m}$. Pipettes 
pulled from borosilicate glass (Hilgenberg, Malsfeld, Germany) with a resistance of 3-7 M $\Omega$ were filled with a solution containing (in $\mathrm{mM}$ ): $140 \mathrm{~K}$-gluconate, $10 \mathrm{HEPES}, 2 \mathrm{MgCl}_{2}, 2 \mathrm{NaATP}$, 10 EGTA, and $0.1 \%$ biocytin, adjusted to $\mathrm{pH} 7.3$ with $\mathrm{KOH}$. Before each experiment, the slice tissue was scanned with the help of laser scanning photo stimulation (Callaway and Katz, 1993) to detect preserved axonal projections. For that, short UV light pulses generated with the help of a continuous wave water-cooled Argon ion laser (ENTC II 652, Coherent, Santa Clara, CA, USA) with two emission lines at 351.1 and $363.6 \mathrm{~nm}$ were flashed to pre-defined locations in the slice. To deliver the light pulses to the tissue, we used our recently developed setup for DPS (Boucsein et al., 2005). Here, light for uncaging is delivered not through the objective of the microscope, but from below the slice through the bottom of the recording chamber (see Figure 1A). Positioning of the laser beam was achieved with the help of two scanning mirrors which were mounted on temperature-stabilized galvanometric scanners (050 EFT, Laser-Scanning-Keiser, Stallikon, Switzerland) driven by appropriate control units (st150). The angular precision of about $1 / 300^{\circ}$ (specification by manufacturer) resulted in a target resolution better than $2 \mu \mathrm{m}$. Laser pulse durations were controlled with the help of a mechanical shutter mounted on an additional scanner unit. The time for settling to a new angular position starting from rest was mainly determined by the inertial mass of the mirrors ( $\tau \approx 1 \mathrm{~ms}$ ) and the shutter plate $(\tau \approx 800 \mu \mathrm{s})$, and thus essentially
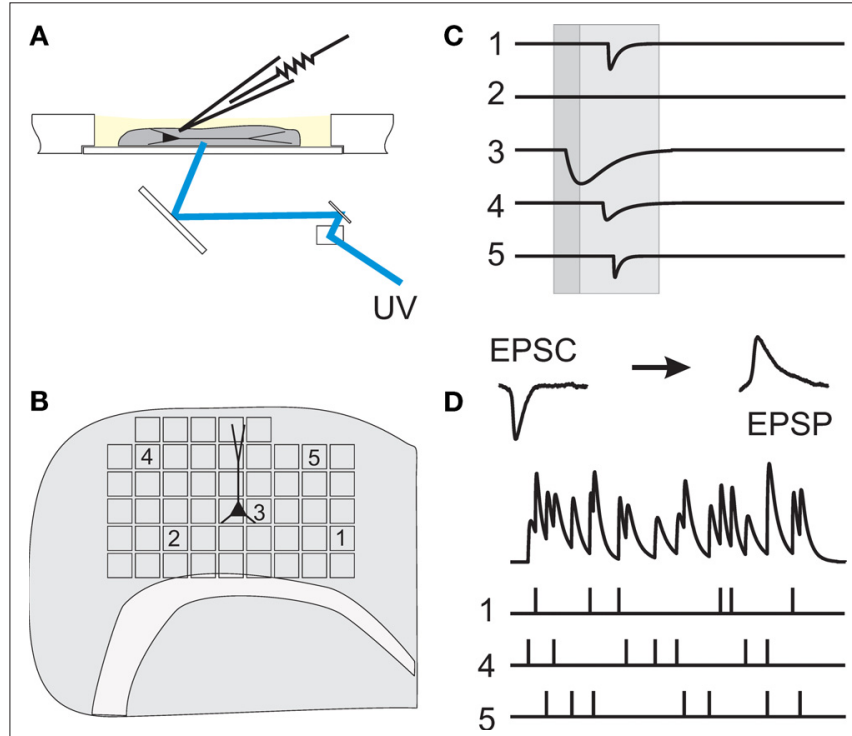

FIGURE 1 | Working principle of dynamic photo stimulation.

(A) Glutamate is released in the tissue from its caged precursor by means of a short light pulse, entering the recording chamber from below. The beam position is controlled by two fast galvanic mirrors. (B) In order to find suitable stimulation sites, i.e. locations within the slice that contain somata of neurons functionally connected to the intracellularly recorded cell, the tissue was scanned with single light pulses applied on a rectangular grid prior to dynamic stimulation. (C) To identify presynaptic locations, postsynaptic current responses to single-shot stimulations are detected in voltage clamp recordings of the postsynaptic cell. (D) To generate spatio-temporal input patterns of synaptic input, presynaptic sites are stimulated in rapid succession, which leads to overlapping postsynaptic potentials in current-clamp recordings. independent of rotation amplitude. To deliver the laser beam to the slice, the tilt-out infrared light condenser (U-SC 2, Olympus, Hamburg, Germany) utilized to establish the electrophysiological recording was swayed backwards and replaced by a mirror mounted at $45^{\circ}$ on a custom-made precision sledge. It deflected the horizontally oriented beam coming from the scanning mirrors vertically towards the bottom of the recording chamber. The laser beam was focused to a spot of about $50 \mu \mathrm{m}$ diameter in the plane of the slice tissue by the use of appropriate zoom optics. To monitor wide regions of the slice, the $40 \times$ water-immersion objective was replaced by a low magnification, long distance objective $(2.5 \times)$. Locations in the slice whose stimulation elicited stimulation-related postsynaptic currents (sPSCs) in the postsynaptic pyramidal cell were collected and, subsequently, either used for the assessment of the physiological parameters of single functional projections, or combined to input trains for the tests of precision and reliability of the signal propagation (Figure 1B-D). In the selection process of presynaptic locations used for our experiments, the following criteria were applied to ensure that only projections from single cells were included in the further analysis: (i) latency of PSC onset from light onset between 15 and 50 ms; (ii) PSC amplitude between 5 and 150 pA; (iii) smooth rising flank of the currents (for details, see Boucsein et al., 2005). Even though application of these criteria substantially reduced the likelihood of using compound PSCs from simultaneously activated connections, we cannot fully exclude this possibility (see also Discussion).

For dynamic stimulation of a high number of presynaptic sites at longer distances from the postsynaptic cell, our setup is advantageous over objective-based systems because it allows rapid beam repositioning $(\approx 1 \mathrm{~ms})$ over a wide spatial range (in our case, covering the entire recording chamber). On the other hand, because of the low numerical aperture of the zoom optics and the use of a UV laser, the release volume is less focused than achievable with an objective and a two-photon laser (Nikolenko et al., 2007). Our method, thus, cannot be used to target the release of caged compounds at a specific depth of the slice tissue, but evokes activity in a rather broad volume within the slice. To estimate the number of cells which get excited by a single laser pulse, a combination of our method with, for example, calcium imaging would be necessary. Coarse estimates suggest that about $30-40$ cells will be illuminated per pulse, of which not all will necessarily fire an action potential.

The quantification of statistical parameters of synaptic events was achieved by repeated single-shot stimulation of the same presynaptic site. In these experiments, inter-shot intervals were taken long enough to fully recover the slice tissue ( $>1 \mathrm{~min}$ ) from glutamate uncaging, i.e. depolarizing responses at presynaptic sites were unchanged throughout repetitions, as tested in a subset of cells by direct photo stimulation of the soma of the patched cell. For testing the reliability of signal integration and transfer in the neocortical sub-network, we dynamically combined activity of as many synapses as possible by generating a time sequence for the activation of the presynaptic neurons (light pulse duration of 3-17 ms) according to a dead-time Poisson process with a mean rate of $20-40 \mathrm{~Hz}$. For each shot, a presynaptic site was randomly drawn from the set of collected sites. Stimulation with this same sequence was then repeated three to nine times and the resulting membrane 
potential fluctuations of the postsynaptic cell were recorded. In most experiments, run-down of the tissue responsiveness to DPS prevented more than 10 repetitions of each sequence.

Electrophysiological signals were recorded with conventional electronics (Axoclamp 2B, Molecular Devices, Sunnyvale, CA), digitized at $20 \mathrm{kHz}$ (CED 1401 Plus, Cambridge Electronic Design, Cambridge, UK), and stored on a computer. Liquid junction potentials were not corrected for. Before digitization, signals were lowpass filtered at $3-5 \mathrm{kHz}$. In addition, voltage clamp recordings were low-pass filtered off-line at $1 \mathrm{kHz}$ for PSC detection. For analysis of inter-trial variability and cross-correlations, we used a bandpass filtered version of the membrane potential $(0.1-200 \mathrm{~Hz})$ and removed line hum by imposing a notch filter (band-stop at $50 \mathrm{~Hz}$ ). Notch filtering was important especially for cross-correlation analysis to exclude artificially high correlations, while band-pass filtering removed artificially low relative variability due to slow drifts in membrane potential, which occurred in some trials. Statistical analyses were performed using Matlab (Mathworks, Natick, MA, USA). After recording, slices were fixed in 4\% paraformaldehyde overnight, and stained with standard procedures (Horikawa and Armstrong, 1988) to confirm cell type and cortical layer of the recorded neuron.

\section{MODELING OF MEMBRANE POTENTIAL FLUCTUATIONS UNDER SYNAPTIC BOMBARDMENT}

We assumed a simple linear model of sub-threshold integration. We defined the number of converging functional connections and the overall PSC frequency based on the average values used in our experiments. Unitary EPSCs were realized as a kernel with the shape of an exponential decay function $\left(\tau_{\mathrm{EPSC}}=5 \mathrm{~ms}\right)$ which, after convolution with the cell's membrane response, results in an EPSP with the shape of the so-called beta function

$\operatorname{EPSP}(t)=\frac{A_{\mathrm{EPSC}}}{\left(C / \tau_{\mathrm{EPSC}}-1 / R\right)} \times\left\{\exp \left[\frac{-t}{(\mathrm{R} \times \mathrm{C})}\right]-\exp \left(\frac{-t}{\tau_{\mathrm{EPSC}}}\right)\right\}$

where average membrane resistance $R=75 \mathrm{M} \Omega$ and membrane capacitance $C=230 \mathrm{pF}$ were estimated from our physiological measurements ( $n=21$ cells). To each simulated functional connection $i$ we randomly assigned a mean amplitude $\mathrm{A}_{i}$ drawn from a lognormal distribution with a geometric mean equal to $31.2 \mathrm{pA}$ and a geometric standard deviation of $1.77 \mathrm{pA}$, as estimated from $n=31$ experimentally probed functional connections (cf. Figure 6A).

\section{STATISTICAL ANALYSIS}

Postsynaptic currents in the membrane current traces of the postsynaptic cell were detected as negative threshold crossings (two to five times the SD of membrane currents measured during the 50 -ms stretch preceding the stimulus). For the analysis of distance dependence of presynaptic locations and PSC amplitudes, lateral distance was measured between the somato-dendritic axis of the postsynaptic cell and the center of the light beam used for uncaging. In a subset of experiments, layers could be clearly assigned in transmission light pictures of slice tissue obtained during the recordings with the help of a low-power objective $(\times 2 / 0.06 \mathrm{NA})$. Layer assignment to presynaptic sites was performed semi-automatically with custom software written in Matlab. To estimate the reliability of stimulation, we measured the percentage of trials that resulted in a detectable EPSC. Variability of PSC amplitude was computed by the coefficient of variation (CV), i.e. the SD of amplitudes measured across repetitions, normalized by the mean amplitude. Temporal precision of the postsynaptic arrival of PSCs was measured as the SD of PSC onset time. Reproducibility of the membrane potential dynamics as recorded during repeated stimulation sequences was evaluated by applying two complementary measures. First, we quantified the normalized variability in signal amplitude between any pair of repetitions, by measuring the ratio of the root mean squared error (RMSE) divided by the mean squared signal amplitude. For any pair $i, j$ of trials, the RMSE of the corresponding voltage traces $\mathrm{V}$ was calculated as the time average:

$\operatorname{RMSE}=\left(\left\langle\left(V_{\mathrm{i}}-V_{\mathrm{j}}\right)^{2}\right\rangle_{t}\right)^{\frac{1}{2}}$.

The root mean squared signal amplitude $\eta$ was calculated as:

$\eta=\left(\left\langle V_{i}^{2}\right\rangle_{t} \times\left\langle V_{j}^{2}\right\rangle_{t}\right)^{\frac{1}{4}}$,

which gives the relative RMSE as:

$\mathrm{RMSE}_{\mathrm{rel}}=\frac{\mathrm{RMSE}}{\eta}$.

To avoid artificially low relative errors, we subtracted baseline membrane potential values from each trial (averaged over $1 \mathrm{~s}$ before stimulation onset). The second measure we used was the reproducibility of any two repetitions expressed as the linear cross-correlation coefficient (CC) of the membrane potential traces. Before averaging the CC across trial pairs, we applied Fisher's $z$-transform.

\section{RESULTS \\ PHYSIOLOGICAL CHARACTERIZATION OF SYNAPSES FORMING INTER-COLUMNAR PROJECTIONS}

The extensive spatial working range of our photo stimulation setup allowed us to scan the entire brain slice in search for intact functional projections. Here, we restricted the scans to regions $<1000 \mu \mathrm{m}$ from the somato-dendritic axis of the patched cell to include intermediate-range projections.

PSCs originating from the activation of synapses were distinguished from direct stimulation of dendritic regions of the postsynaptic cell by their longer latencies and faster rise times (Figure 2A). Assessment of the lateral distance of presynaptic sites to the somatodendritic axis of the recorded cells showed that we, indeed, collected many synapses at intermediate distances from different layers, with a bias towards synapses from the infragranular layers (Figures 2B,C). PSC amplitude did not seem to depend strongly on lateral distance from the postsynaptic cell (Figure 2D).

Before assessing the physiological parameters of the synaptic connections, we reconfirmed the reliability of our method to elicit action potentials in the presynaptic cells. For that, we randomly patched cells throughout the slice and stimulated them with glutamate released directly at the soma. This stimulation reflected the situation which was, later on, generated during the scans of the putative presynaptic cells. Direct somatic stimulation of cells revealed that, if stimulation was chosen to be strong enough to elicit an action potential, subsequent shots with the same parameters also generated action potentials with a reliability of $100 \%$ $(n=14$; Figure 3A). 


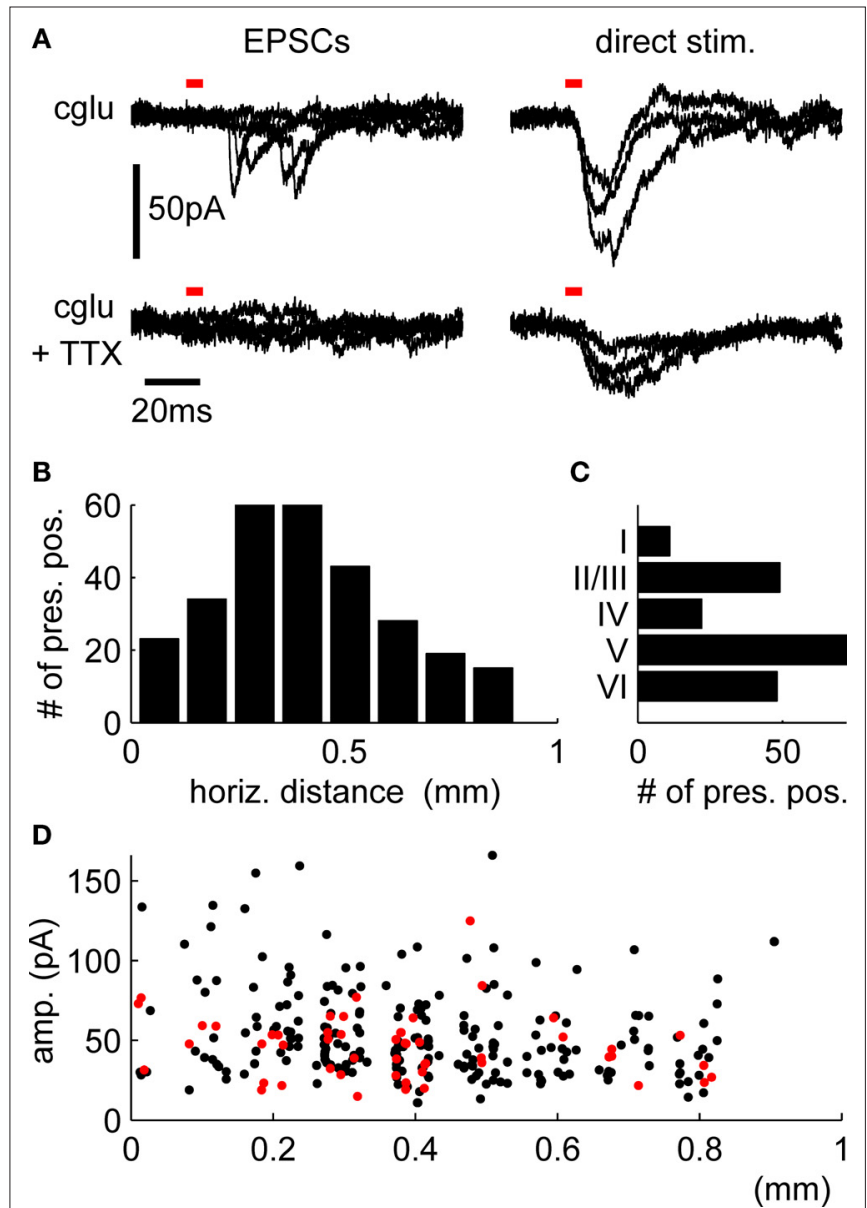

FIGURE 2 | Confirmation of the presynaptic origin of postsynaptic currents and distribution of presynaptic locations. (A) Postsynaptic currents from stimulation of presynaptic cells (left panels) show faster rise times and longer latencies to stimulus onsets than those from direct stimulation of the dendritic regions by uncaged glutamate (right panels). Repeated stimulation after incubation with TTX confirms the presynaptic origin of the PSCs shown in the upper left panel, while direct responses remain (lower right panel). Red bars indicate light pulse duration, traces are overlays from four presynaptic and three direct stimulation sites, respectively. Distribution of total numbers of synapses over the horizontal distance (B) and layers (C) shows a bias towards intermediate-range projections from the infragranular layers. Local connections are underrepresented because we excluded the PSCs confounded with direct responses from our analysis (D) Distance dependence of PSC amplitudes for all synapses used for the quantification of synaptic physiology (red dots; cf. Figure 3) and for the dynamic stimulations (black dots; cf. Figures 4 and 5).

Next, we assessed the reliability of synaptic transmission, temporal precision of PSC formation and amplitude variation by measuring PSCs by repeated activation of the same projection. In general, special care was taken to sort out all presynaptic locations whose illumination led to direct dendritic stimulation of the postsynaptic cell. These currents from direct stimulation would not reflect synaptic physiology and, thus, distort the results of the study. As has been shown previously (Boucsein et al., 2005), direct stimulation activates currents with symmetrical rise- and decay times, which cannot be blocked by application of TTX (Figure 2A). This selection criterion led to the exclusion of projections from cells in close

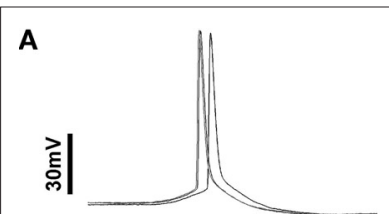

B
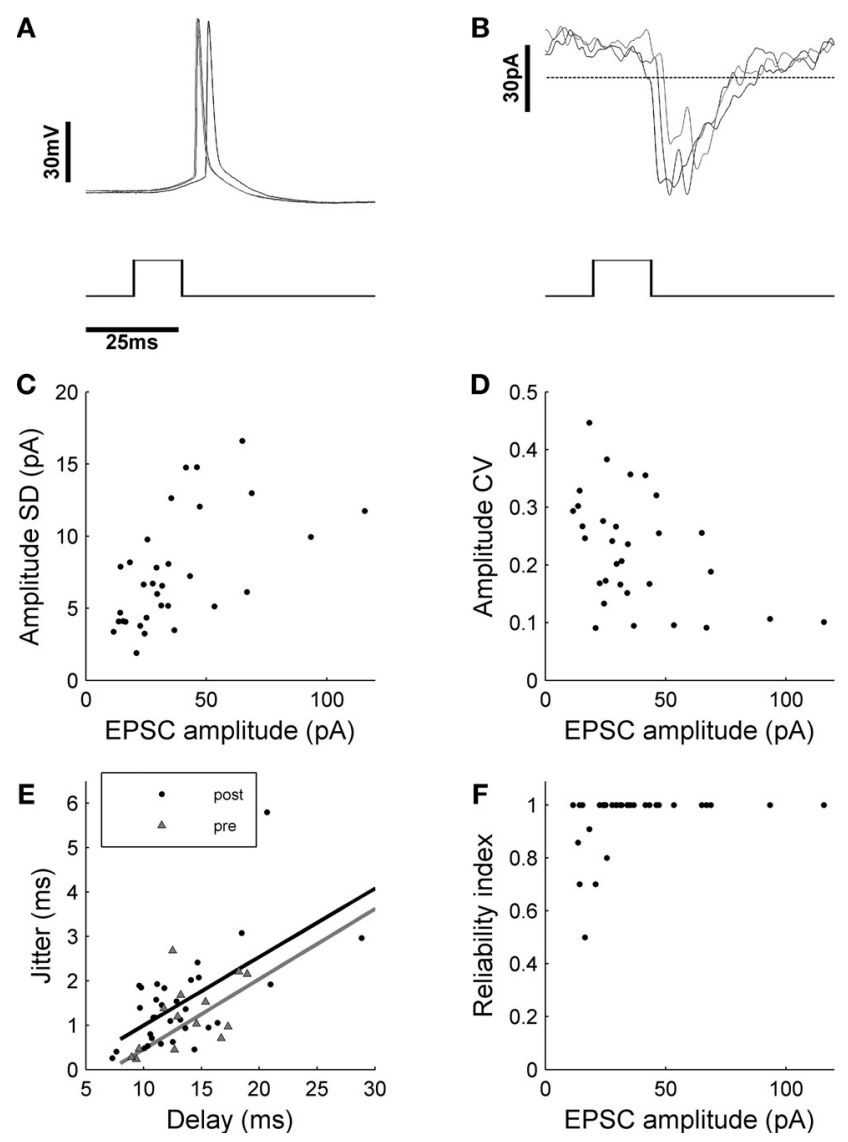

FIGURE 3 | Quantification of synaptic variability. (A) Single-shot stimulation of presynaptic cells evoked action potentials with a reliability of 100\% ( $n=14$ cells). (B) In the postsynaptic cell, isolated PSCs could be detected, which were quantified in terms of absolute and relative amplitude variability (C,D). The long absolute latencies of up to $30 \mathrm{~ms}$ after stimulus onset, which became apparent when temporal jitter of PSC onset (filled circles) was evaluated (E), originated largely from the presynaptic spike formation, as tested with direct shots to the somata of cells patched in different layers of the slice tissue (grey triangles). Fitting linear regression lines to the data points representing either pre- or postsynaptic events, showed that latency jitter was mainly due to the process of presynaptic spike formation. Actual temporal jitter of synaptic transmission could be estimated as the difference in intercept of the linear regression lines with the $y$-axis, which yields a low temporal jitter of $0.57 \mathrm{~ms}$. (F) Reliability of synapses was very high, possibly because of multiple synapses per cell-to cell connection.

proximity to the dendritic region of the postsynaptic cell, since they were always confounded with direct stimulation responses. For the assessments of the physiological properties of synaptic connections, each functional connection was tested separately by applying a single light pulse to the corresponding presynaptic location. For these tests, we used eight cells with a total of 32 presynaptic locations whose stimulation lead to clear EPSCs. Depending on the stability of the recording and the number of presynaptic sites tested, this stimulation was then applied repeatedly 4-11 times, resulting in a number of similar responses (overlay plot for one example shown in Figure 3B). Statistical analysis of the respective data revealed a moderate amplitude variability of PSCs elicited with DPS (Figures 3C,D; mean CV of $0.24 \pm 0.11$ ), which agreed well 
with previous findings from other studies (Feldmeyer et al., 1999; Markram et al., 1997; Mason et al., 1991). More strikingly, a high temporal precision of the responses (Figure 3E) and a surprisingly high reliability (close to $100 \%$; Figure 3 F) was found.

A puzzling finding was the latency distribution of the onset of the PSCs with a range from a few up to $\sim 30 \mathrm{~ms}$, the time between the UV light flash and PSC onset was much too long to be explained by axonal delays alone (1-2 ms in un-meylinated axons in the cortex). Assessment of the delays between stimulus and spike onset in the recordings from the candidate presynaptic cells stimulated at the soma revealed that the observed long latencies and the correlation of latency and temporal jitter found in PSCs was, in fact, due to the process of driving the presynaptic cell to fire a spike. Interestingly, the latency jitter of the PSCs is, in general, slightly higher than for the presynaptic spikes (Figure 3E). The difference between the two lower bounds can be attributed to the actual jitter in synaptic transmission and was estimated to be very small with $\sim 0.5 \mathrm{~ms}$. This number would even be smaller for bias-free estimates without measurement noise. In summary, synaptic transmission occurred with a reliability of close to $100 \%$, amplitude variability of $24 \pm 10 \%$, and at temporal jitter of $<1 \mathrm{~ms}$.

\section{REPRODUCIBILITY AND PRECISION OF SIGNAL INTEGRATION}

To assess how reproducibly signals from intermediate-range projections are propagated to and integrated in pyramidal layer 5 neurons, we dynamically combined the stimulation of all presynaptic cells obtained from the initial tissue scan. For these tests, we used a second set of cells $(n=18)$ where we could find at least seven presynaptic locations whose stimulation led to clear EPSC's (average 15.7 locations per cell). The short exposure times which were needed to elicit presynaptic spikes (3-6 ms) and the fast scanning speed of our setup ( $<1 \mathrm{~ms}$ for beam re-positioning) allowed for input rates of up to $200 \mathrm{~Hz}$. Avoiding presynaptic locations which also led to direct stimulation of the postsynaptic cell ensured that the complete input train onto the postsynaptic cell was composed of synaptic events only, including a presynaptic spike, activation of the presynaptic terminal, vesicle release and opening of postsynaptic ligand-gated ion channels. Application of the same input train under TTX $(4 \mu \mathrm{M})$, which was performed in most experiments (15 out of 18 ), confirmed that our selection criterions were appropriate: in none of these control experiments, a postsynaptic potential could be detected (see Figure 4).

After generating a stimulation sequence employing the selected presynaptic sites (see Materials and Methods), we applied this same sequence three to five times to the slice tissue, while monitoring the membrane potential fluctuations of the recorded cell (Figure 4). Visual comparison of traces from individual trials revealed a remarkable reproducibility across trials. To quantify the differences between individual trials, we used two different measures, emphasizing two different aspects of the variability of the response (cf. Materials and Methods). First, we determined the amplitude variation of the membrane potential fluctuations by measuring the relative variation $\left(\mathrm{RMSE}_{\mathrm{rel}}\right)$. In a total of $n=18$ experiments we measured an average inter-trial variability of membrane potential fluctuation amplitudes of $58.2 \pm 13 \%$ for the $\mathrm{RMSE}_{\text {rel }}$ (range 38-90\%). Second, we measured the linear CC to quantify the similarity of the temporal structure of the fluctuation waveforms in different trials. The voltage traces,

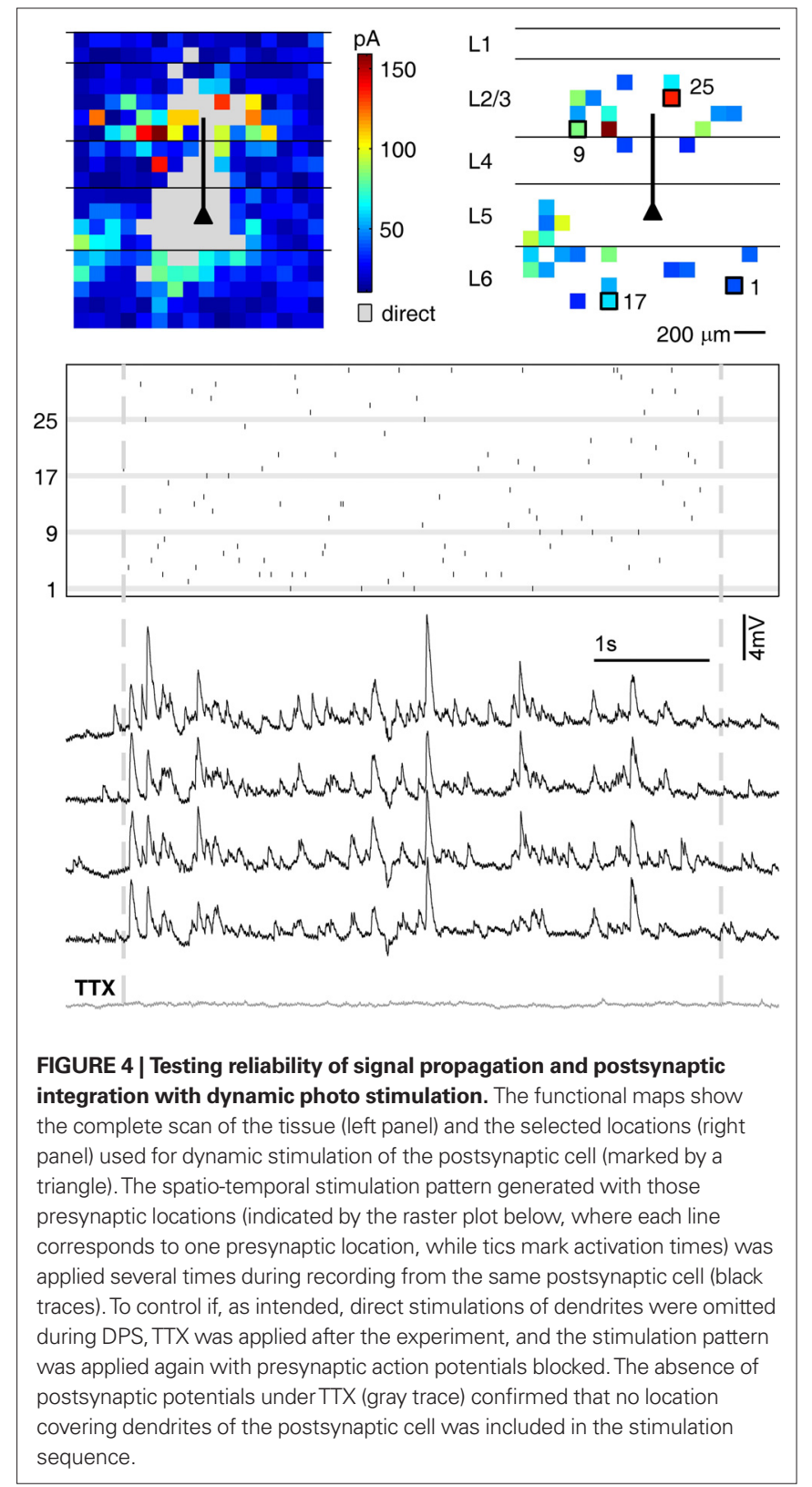

as those in Figure 4, exhibited a high average trial-to-trial correlation, indicating a high temporal precision that was preserved across repeated stimulation runs despite the considerable amplitude variability. The trial-to-trial correlation across all experiments was on average CC $=0.83$ (range 0.54-0.96).

To estimate how both measures of amplitude variability and of waveform reproducibility are influenced by the common noise of the experimental system, we compared inter-trial variability with inter-realization variability in an additional experiment where we generated two different realizations of a stimulation sequence using identical statistical parameters but visiting the presynaptic sites in different order and at different times. Both realizations were applied to the slice tissue three times each, and the overlay of voltage traces from the same realizations again displays a high similarity (Figure 5A). 
Averaged inter-trial cross-correlograms between trials from the same realization (Figure 5B) indicated that similarity of the temporal structure of the responses was high on an extended time-scale. By contrast, comparison between trials from different realizations revealed little similarities in membrane potential traces, except for the mean amplitude and approximate total charge which is proportional to the area under the curves. Quantification of amplitude variability across different stimulation patterns yielded an average $\mathrm{RMSE}_{\text {rel }}=190.0 \pm 21.2 \%$, which is much higher than the inter-trial variability within the same stimulation pattern $(48.2 \pm 10.3 \%$; avg. for both patterns). As expected, correlation among membrane potential traces that stem from different input patterns dropped to low values of $\mathrm{CC}=0.16 \pm 0.03$ (cf. Figures 5C,D).

\section{NUMERICAL SIMULATIONS OF DIFFERENT SOURCES OF VARIABILITY}

To evaluate which source - amplitude variance of synaptic transmission, latency jitter or noisiness of the dendritic integration mechanism - contributed most to the measured trial-to-trial variability, we designed a simple model of synaptic integration (see Materials and Methods), using the physiological parameters (EPSC amplitude distribution and temporal jitter) measured during activation of single synapses (Figure 3 ).

Into this model, we fed input spike trains with the same statistical parameters as used in our first set of experiments and, again, measured trial-to-trial variability with the help of the $\mathrm{RMSE}_{\text {rel }}$ and CC (Figure 6). Since spontaneous EPSPs as well as the measurement noise of the electronic equipment are expected to have some impact on the response variability, we cut out data stretches from the patchclamp recordings prior to DPS and added them to the simulated PSP traces. When quantifying the relative amplitude error as well as the $\mathrm{CC}$ for different values of PSC amplitude variability $\left(\mathrm{CV}_{\mathrm{A}}\right.$ between 0.1 and 0.8 ) and zero latency jitter, we found that the trial-to-trial error in amplitude showed a strong dependence on $\mathrm{CV}_{\mathrm{A}}$ (Figure 6E). By contrast, the linear correlation coefficient, which measures the temporal trial-to-trial similarity, was still high for increased synapse variability (Figure 6F). The observed variations in latency jitter of the synaptic events, estimated to be $<1 \mathrm{~ms}$ (Figure $3 \mathrm{E}$ ), hardly influenced $\mathrm{RMSE}_{\mathrm{rel}}$ and CC (data not shown).
A
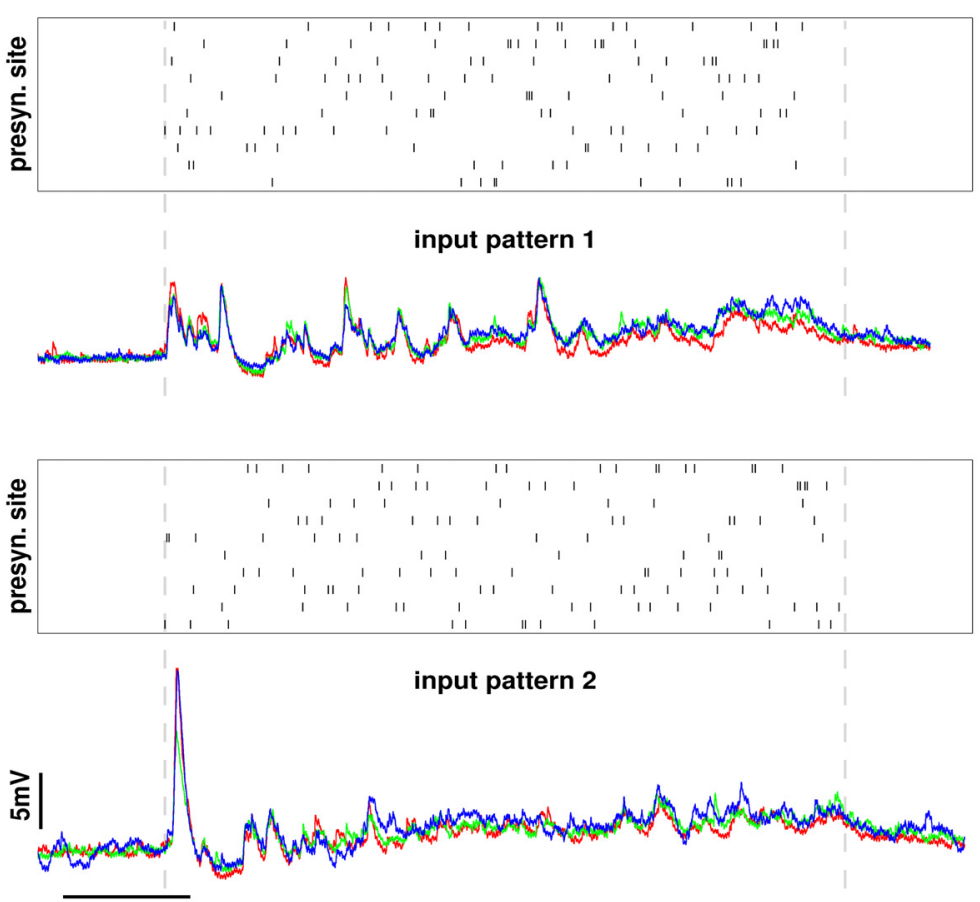

input pattern 2

1s

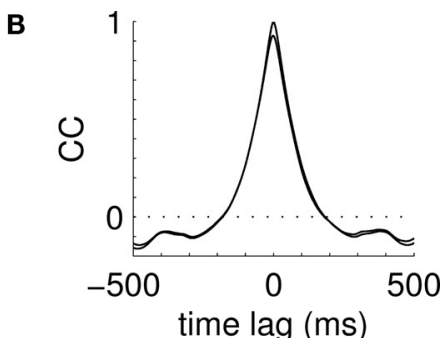

C

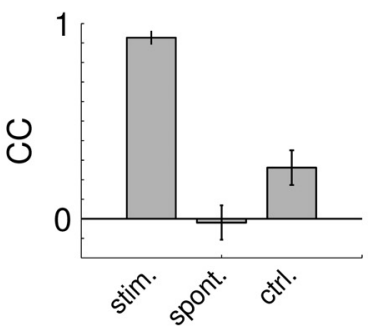

D

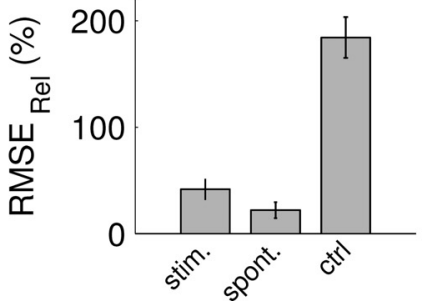

FIGURE 5 | Estimating the influence of the experimental system on $\mathbf{R M S E}_{\text {ret }}$ and CC measures. The same postsynaptic cell was repeatedly stimulated with two distinct spatio-temporal patterns, and, subsequently, $\mathrm{RMSE}_{\mathrm{re|}}$ and CC were quantified across trials of the same and across trials of different patterns. (A) Raster plots indicate spatio-temporal activation of first (upper) and second (lower) input pattern. The overlay of membrane potential traces below show that each stimulation pattern reliably reproduced the waveform of membrane potential fluctuation over three consecutive trials, while the voltage traces differed strongly for the different patterns. (B) The average cross-correlation function from pairings of trials of the same stimulation pattern (average over two patterns à three trials) in black is almost identical to the average auto-correlation function (grey trace). (C) The average linear correlation of membrane potential traces across trials was very high for the same stimulation pattern (stim) with $\mathrm{CC}=0.92 \pm 0.01$ (pattern 1) and $0.90 \pm 0.02$ (pattern 2) but low across trials that stem from different stimulation patterns $(0.16 \pm 0.03$; ctrl). Spontaneous activity (spont) served as an additional control showing residual cross-trial correlation close to 0 . (D) Amplitude variability was considerable across trials of the same stimulation pattern (stim) with $\mathrm{RMSE}_{\text {rel }}=40.4 \pm 6.1$ (pattern 1) and $56 \pm 3.9 \%$ (pattern 2), but much higher when measured across trials from different stimulation patterns $(190.0 \pm 21.2 \%$; ctrl). Stimulation parameters for both input patterns: pulse duration $4 \mathrm{~ms}$, stimulation rate $25 \mathrm{~Hz}$, total of 125 stimulation pulses. 


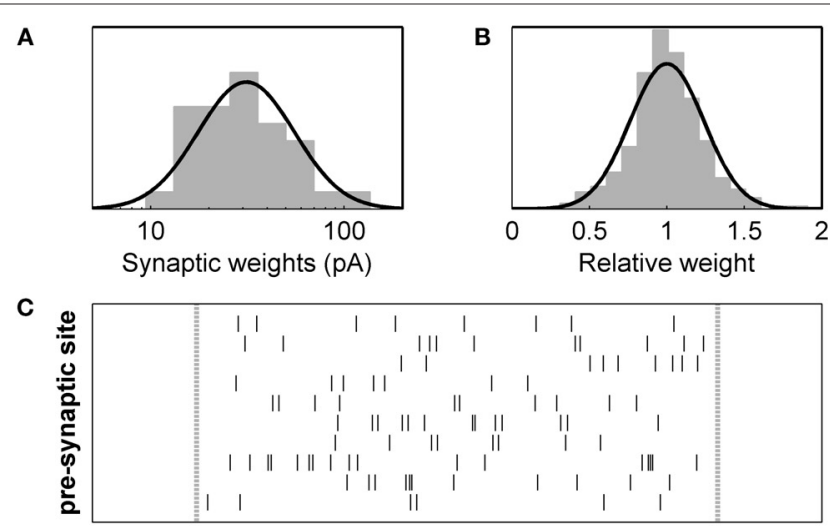

D

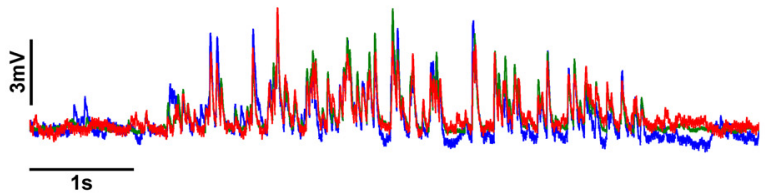

E

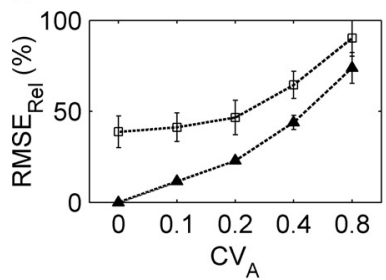

$\mathbf{F}$

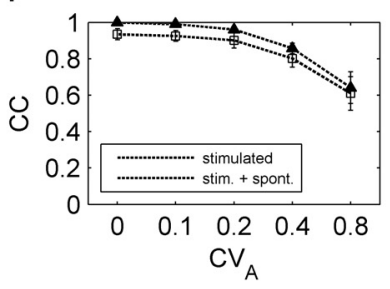

FIGURE 6 | Estimation of contributions of different sources of variability with the help of a linear model of synaptic integration.

(A) Weights of individual synapses were randomly pulled from a log-normal distribution fitted to the data from the single-shot experiments, and the amplitude of each modelled synapse was varied form trial to trial according to a Gaussian fit to the relative amplitude distribution of the same data (B). With these synapses, input patterns were simulated corresponding to those generated experimentally (C), and the resulting simulated membrane potential fluctuations (D) were analyzed in the same way. Varying the amplitude variability of the simulated synapses revealed that, with increasing $\mathrm{CV}_{\mathrm{A}^{\prime}}$ amplitude variability of the membrane potential fluctuations also increased markedly (E) while the $\mathrm{CC}$ decreased notably only for large values of $\mathrm{CV}_{\mathrm{A}}(\mathbf{F})$.

How much of the experimentally measured amplitude variability can be explained by combining the known sources of noise in our model? To test this, we matched each individual experiment with a model simulation using the appropriate average stimulation parameters and spontaneous membrane potential traces from the very same neuron. The model parameters were chosen in accordance with our experimentally used average presynaptic activation rate $(28 \mathrm{~Hz})$ and average number of presynaptic sites $(n=16)$. They also conformed to the measured variability (see Figure 3), i.e. average synaptic amplitude variability of $\mathrm{CV}_{\mathrm{A}}=0.24$ and latency jitter (including presynaptic jitter of spike initiation) with an average standard deviation of $1.48 \mathrm{~ms}$. Comparison to the experimental results (Figure 7) revealed that synapse variability explains about half of the experimentally observed response variability.

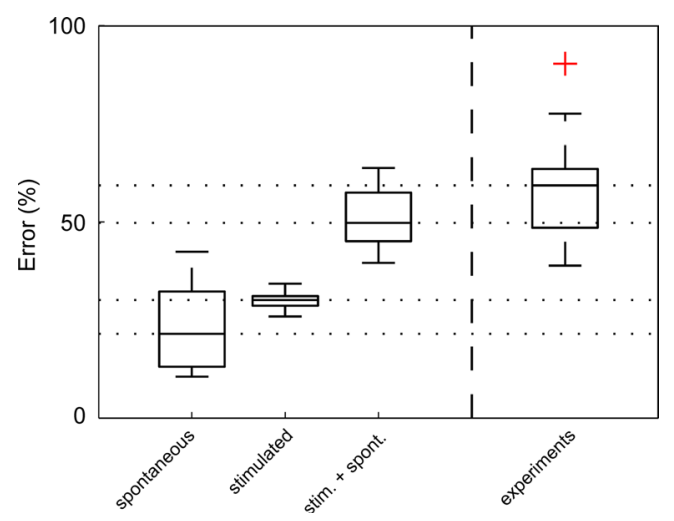

FIGURE 7 | Most of the experimentally observed inter-trial variability can be explained by synapse amplitude variability, spontaneous events and measurement noise. The average $(n=18) \mathrm{RMSE}_{\mathrm{rel}}$ of inter-trial variability of spontaneous activity alone (including measurement noise), derived from experimentally recorded membrane potential fluctuations prior to stimulation, was calculated to be $22 \%$ (spontaneous), which is approximately half of the experimentally measured RMSE rel $_{\text {(experiments). }}$ Simulated membrane potential fluctuations with realistic synaptic variability values alone (without noise) produced a RMSE $\mathrm{rel}_{\text {of }} 27 \%$ (stimulated). When spontaneous activity - including spontaneous presynaptic events, spontaneous releases and measurement noise - was added $\left(\mathrm{RMSE}_{\mathrm{rel}}=51 \%\right)$, more than $85 \%$ of the average amplitude variability was explained. Red cross denotes one outlier.

\section{DISCUSSION}

In the present study, we characterized failure rate, physiological strength, temporal precision and amplitude variability of intermediate-range projections onto layer 5 pyramidal cells of the rat neocortex, and, subsequently, used these projections to dynamically test the reliability and precision of signal integration in cortical inter-columnar sub-networks. Intermediate-range connections were surprisingly reliable and showed moderate amplitude variability. According to the numerical simulations in our simple model of dendritic integration, the synaptic variability accounted for most of the amplitude variability of membrane potential fluctuations observed under dynamic stimulation of several synapses. Synaptic physiology, thus, largely determines amplitude variability and temporal precision of activity propagation through intermediate-range projections, at least in an activity regime with low firing rates. Based on our results we hypothesize that cortical neurons are optimized to reliably detect and transmit coincident inputs or, likewise, fast transients of ensemble input rate and to produce reliably timed action potentials. This suggests that neocortical networks can operate in a temporally highly precise regime, which is the prerequisite for spike-time based coding strategies.

\section{ARE INTERMEDIATE-RANGE SYNAPSES OPTIMIZED FOR RELIABLE TRANSMISSION?}

The high reliability of synaptic connections onto layer 5 pyramidal neurons observed in our data are somewhat surprising: even though reliability of strong synapses between neocortical cells has been reported to be high (for the strongest synapses close to 100\%; Markram et al., 1997), weaker synapses, which were reported 
to dominate the range between 50 and $250 \mu \mathrm{m}$ lateral distance between pre- and postsynaptic cells in layer 2/3 (Holmgren et al., 2003), seem to be much less reliable. As the statistical analysis of the intermediate-range projections from our experiments shows, there is no marked drop in PSC amplitude with increasing distance, a finding that has also been described recently for local synapses (Matsuzaki et al., 2008). The question, whether the intermediaterange projections are especially strengthened during development, i.e. by learning processes or selective pruning of weak synapses, is difficult to answer from our data, since we cannot exclude the possibility of having missed the weak connections with our PSC detection method. Nevertheless, the mere fact that there are so many strong connections is noteworthy, since it suggests that they can heavily influence the discharge of the postsynaptic neuron and, thus, play an important role in information processing within the neocortex.

In general, the conclusions drawn from our data largely depend on the assumption that with DPS we activated only one cell with each single light pulse that projected onto the postsynaptic cell, and that there were no polysynaptic pathways involved. In how far this assumption is justified cannot be answered unequivocally without the actual identification and intracellular stimulation of the photo-activated, presynaptic cells, a technical challenge that has recently been described to be achievable with the help of combined photostimulation and calcium imaging (Nikolenko et al., 2007). Due to optical limitations, though, it is still not possible to use such setups for dynamic photostimulation of intermediate-range projections, since the spatial working range is limited by the highpower objectives necessary for focusing the stimulating light beam. The assumption that a single light flash activates monosynaptic responses from single cells seems to be valid in some experimental setups employing laser uncaging of glutamate in acute brain slices (Weiler et al., 2008) while in others, multiple cells seem to be activated frequently with every flash (Schubert et al., 2003). These differences are most likely due to the technical details such as the specific light source, focusing, and minimal exposure time. Two findings in our data support that in our setup usually only one presynaptic cell gets activated. First, the connection probability in the distance range we used for stimulation is very low (Hellwig, 2000; Song et al., 2005; Thomson and Bannister, 2003), making it unlikely that two neighboring cells are connected to the same postsynaptic cell. Second, as was shown by the direct stimulation of putative presynaptic cells (Figure 3), latencies from light pulse to spike onset scattered over $30 \mathrm{~ms}$ between cells. Since we detected connections as postsynaptic PSCs, which showed rise times in the low millisecond range, combined PSCs from two neighboring presynaptic cells hit by the same light pulse could have only be misinterpreted as the projection from a single cell if the latencies from pulse onset to spike onset of both cells were more or less identical, which we consider a rather unlikely scenario. Otherwise, the overlapping PSCs would have been easily detected as compound PSCs with two or more separable rising flanks (Boucsein et al.2005). In our mapping data, we only rarely found potential candidates for compound PSCs. These ambiguous responses were, in any case, excluded from further stimulation protocols. Experiments that include the identification of the presynaptic cells as well as the morphological reconstruction of their axonal arborizations and putative synaptic contacts to the postsynaptic cell could provide information about the cellular mechanisms which make intermediate-range connections reliable. Both, multaptic connections, which have been previously described for closely neighboring neocortical cells (Koester and Johnston, 2005; Markram et al., 1997; Tamas et al., 2002), or single synapses with high release probability could provide physiological means to enhance the reliability of connections between cells (Silver et al., 2003).

\section{INPUT RATES IN VITRO COMPARED TO IN VIVO}

The assessment of the dynamic integration behavior of pyramidal cells in our experimental setup is limited by the maximum stimulation rate of presynaptic sites. With DPS, we are currently able to produce input rates of $\sim 50 \mathrm{spikes} / \mathrm{s}$, distributed over up to 40 individual functional contacts. The question how realistic these input rates are for the evaluation of the situation in the intact brain is difficult to answer. Firing rates of individual cells in cat visual cortex can reach more than $100 \mathrm{~Hz}$ during sensory stimulation (Carandini and Ferster, 2000; Hubel and Wiesel, 1962). In rat barrel cortex, on the other hand, average firing rates in vivo ranging from $0.5 \mathrm{~Hz}$ (Lee et al., 2006; Manns et al., 2004) to $0.05 \mathrm{~Hz}$ (Brecht and Sakmann, 2002; Margrie et al., 2002; Waters and Helmchen, 2006) have been described. Pyramidal cells in monkeys have been estimated to carry $\sim 20.000$ synapses (Abeles, 1991), while the number of synapses per layer 5 pyramidal neuron in the rat neocortex are more likely to be in the range of 10.000-12.000 (Warren and Bedi, 1982), of which approximately one-half is located on the tuft of the distal apical dendrite (Binzegger et al., 2004; Warren and Bedi, 1982), where they most likely form a separate integration unit and have only limited direct effects on somatic integration (Berger et al., 2003; Larkum et al., 1999; Williams and Stuart, 2002). In conclusion, when considering 5.000 synapses near the soma and an average firing rate of $0.05 \mathrm{~Hz}$ as measured in rat barrel cortex, the rate of synaptic inputs of 50 spikes/s achievable with DPS in acute slices might at least reach into the lower range of realistic input rates for the in vivo situation in certain brain areas.

\section{IMPLICATIONS FOR THEORIES OF CORTICAL CODING}

When we interpret our results in the context of a classical rate code, where the mean number of spikes per unit time carries the information, but where spikes are otherwise positioned randomly in time, and if we assume that the number of inputs is fixed over repeated trials, i.e. zero input noise, the observed output variability will be mainly a consequence of the variability of EPSC amplitude. Even for this noise-free input scenario we must expect that the modest EPSC amplitude variability will be translated into a considerable trial-to-trial output variability in the number of spikes per unit time (Carandini, 2004). Thus, the observed trialto-trial variation of synaptic amplitude as well as additional small trial-to-trial variation of the overall input rates due to ongoing processing (Arieli et al., 1996; Azouz and Gray, 1999; Kisley and Gerstein, 1999; Nawrot et al., 2008) contributes significantly to a noisy output rate signal. The neocortical sub-network consisting of intermediate-range projections, as described in the present study would, thus, not be very suitable to reliably transmit firing rates (see Kumar et al., 2008 for additional evidence from a recent modeling study). 
On the other hand, our results suggest that a spike timing based coding strategy is feasible, given that the presynaptic network assures appropriate temporal input structure. Our experiments demonstrated (i) a synaptic transmission that is temporally precise in the sub-millisecond regime, and (ii) a single cell integration of synaptic input that preserves the high temporal precision of the synaptic input. Thus, a cortical layer 5 neuron can reliably convert temporally structured presynaptic input into fast dynamic membrane potential deflections, and finally translate these into precisely timed action potential output due to its reliable spike generating mechanism (e.g. Mainen and Sejnowski, 1995). Together with known mechanisms of reducing the effective time constant, dynamic threshold adaptation, and feed-

\section{REFERENCE}

Abeles, M. (1991). Corticonics: Neural Circuits of the Neocortex. Cambridge, Cambridge University Press.

Alonso, J.M., Usrey, W.M., and Reid, R. C., (1996). Precisely correlated firing in cells of the lateral geniculate nucleus. Nature 383, 815-819.

Arieli, A., Sterkin, A., Grinvald, A., and Aertsen, A. (1996). Dynamics of ongoing activity: explanation of the large variability in evoked cortical responses. Science 273, 1868-1871.

Azouz, R., and Gray, C. M. (1999). Cellular mechanisms contributing to response variability of cortical neurons in vivo. J. Neurosci. 19, 2209-2223.

Berger, T., and Luscher, H. R. (2003). Timing and precision of spike initiation in layer $\mathrm{V}$ pyramidal cells of the rat somatosensory cortex. Cereb. Cortex 13, 274-281.

Berger, T., Senn, W., and Luscher, H. R. (2003). Hyperpolarization-activated current $I_{\mathrm{h}}$ disconnects somatic and dendritic spike initiation zones in layer V pyramidal neurons. J. Neurophysiol. 90, 2428-2437.

Binzegger, T., Douglas, R. J., and Martin, K. A. (2004). A quantitative map of the circuit of cat primary visual cortex. J. Neurosci. 24, 8441-8453.

Boucsein, C., Nawrot, M., Rotter, S., Aertsen, A., and Heck, D. (2005). Controlling synaptic input patterns in vitro by dynamic photo stimulation. J. Neurophysiol. 94, 2948-2958.

Boucsein, C., Tetzlaff, T., Meier, R., Aertsen, A., and Naundorf, B. (2009). Dynamical response properties of neocortical neuron ensembles: multiplicative versus additive noise. J. Neurosci. 29, 1006-1010.

Braitenberg, V. (1978). Cell assemblies in the cerebral cortex. In Theoretical Approaches to Complex Systems, R. Heim and G. Palm, eds (Berlin, Springer), pp. 171-188.

Brecht, M., and Sakmann, B. (2002). Dynamic representation of whisker deflection by synaptic potentials in spiny stellate and pyramidal cells in the barrels and septa of layer 4 rat somatosensory cortex. J. Physiol. (Lond.) 543, 49-70.

Buzas, P., Kovacs, K., Ferecsko, A.S. Budd, J. M., Eysel, U. T., and Kisvarday, Z. F. (2006). Model-based analysis of excitatory lateral connections in the visual cortex. J. Comp. Neurol. 499, 861-881.

Callaway, E. M., and Katz, L. C. (1993). Photostimulation using caged glutamate reveals functional circuitry in living brain slices. Proc Natl Acad Sci U.S.A. 90, 7661-7665.

Carandini, M. (2004). Amplification of trial-to-trial response variability by neurons in visual cortex. PLoS Biol. 2, E264. Membrane potential and firing rate in cat primary visual cortex. J. Neurosci. 20, 470-484.

Cox, C. L., Denk, W., Tank, D. W., and Svoboda, K. (2000). Action potentials reliably invade axonal arbors of rat neocortical neurons. Proc Natl Acad Sci U.S.A. 97, 9724-9728.

Debanne, D., Gahwiler, B. H., and Thompson, S. M. (1996). Synaptic and non-synaptic plasticity between individual pyramidal cells in the rat hippocampus in vitro. J. Physiol. (Paris) 90, 307-309.

Diesmann, M., Gewaltig, M. O., and Aertsen, A. (1999). Stable propagation of synchronous spiking in cortical neural networks. Nature 402, 529-533.

Feldmeyer, D., Egger, V., Lubke, J., and Sakmann, B. (1999). Reliable synaptic connections between pairs of excitatory layer 4 neurones within a single 'barrel' of developing rat somatosensory cortex. J. Physiol. 521.1, 169-190.

Frick, A., Feldmeyer, D., Helmstaedter, M., and Sakmann,B.(2008). Monosynaptic connections between pairs of L5A pyramidal neurons in columns of juvenile rat somatosensory cortex. Cereb. Cortex 18, 397-406.
Carandini, M., and Ferster, D. (2000).

forward inhibition, and in view of previous findings suggesting an increased efficacy of synchronous inputs on postsynaptic spike initiation (Alonso et al., 1996), our results provide additional evidence that cortical networks contain cellular structures that are well suited for temporal coding schemes that rely on precise spike timing.

\section{ACKNOWLEDGMENTS}

This project received funding from the German Federal Ministry of Education and Research, grant 01GQ0413 to BCCN Berlin and grant 01GQ0420 to BCCN Freiburg, as well as from the European Union (EU Grant 15879, FACETS) and from the German Research Council (DFG-SFB 780).

Gil, Z., Connors, B. W., and Amitai, Y. (1999). Efficacy of thalamocortical and intracortical synaptic connections: quanta, innervation, and reliability. Neuron 23, 385-397.

Harris, K. D. (2005) Neural signatures of cell assembly organization. Nat. Rev. Neurosci. 6, 399-407.

Hellwig, B. (2000). A quantitative analysis of the local connectivity between pyramidal neurons in layers $2 / 3$ of the rat visual cortex. Biol. Cybern. 82, 111-121.

Holmgren, C., Harkany, T., Svennenfors, B., and Zilberter, Y. (2003). Pyramidal cell communication within local networks in layer $2 / 3$ of rat neocortex. J. Physiol. 551, 139-153.

Horikawa,K., and Armstrong,W.E.(1988) A versatile means of intracellular labeling: injection of biocytin and its detection with avidin conjugates. J. Neurosci. Methods 25, 1-11.

Hubel, D. H., and Wiesel, T. N. (1962). Receptive fields, binocular interaction and functional architecture in the cat's visual cortex. J. Physiol. 160, 106-154.

Kisley, M. A., and Gerstein, G. L. (1999) Trial-to-trial variability and statedependent modulation of auditoryevoked responses in cortex. J. Neurosci. 19, 10451-10460.

Koester, H. J., and Johnston, D. (2005) Target cell-dependent normalization of transmitter release at neocortical synapses. Science 308, 863-866.

Köndgen, H., Geisler, C., Fusi, S., Wang, X. J., Luscher, H. R., and Giugliano, M. (2008). The dynamical response properties of neocortical neurons to temporally modulated noisy inputs in vitro. Cereb. Cortex 18, 2086-2097.

Konig, P., Engel, A. K., and Singer, W. (1996). Integrator or coincidence detector? The role of the cortical neuron revisited. Trends Neurosci. 19, 130-137.

Kumar, A., Rotter, S., and Aertsen, A. (2008). Conditions for propagating synchronous spiking and asynchronous firing rates in a cortical network model. J. Neurosci. 28, 5268-5280.

Larkum, M. E., Zhu, J. J., and Sakmann, B. (1999). A new cellular mechanism for coupling inputs arriving at different cortical layers. Nature 398, 338-341.

Lee, A. K., Manns, I. D., Sakmann, B., and Brecht, M. (2006). Whole-cell recordings in freely moving rats. Neuron 51 , 399-407.

Losonczy, A., and Magee, J. C. (2006). Integrative properties of radial oblique dendrites in hippocampal CA1 pyramidal neurons. Neuron 50, 291-307.

Lüscher, C., Streit, J., Lipp, P., and Luscher, H. R. (1994). Action potential propagation through embryonic dorsal root ganglion cells in culture. II. Decrease of conduction reliability during repetitive stimulation. J. Neurophysiol. 72, 634-643.

Magee, J.C. (2000). Dendritic integration of excitatory synaptic input. Nat. Rev. Neurosci. 1, 181-190.

Mainen, Z. F., and Sejnowski, T. J. (1995). Reliability of spike timing in neocortical neurons. Science 268 , 1503-1506.

Manns, I. D., Sakmann, B., and Brecht, M. (2004). Sub- and suprathreshold receptive field properties of pyramidal neurones in layers $5 \mathrm{~A}$ and $5 \mathrm{~B}$ of rat somatosensory barrel cortex. J. Physiol. 556, 601-622.

Margrie, T. W., Brecht, M., and Sakmann, B. (2002). In vivo, lowresistance, whole-cell recordings from neurons in the anaesthetized and awake mammalian brain. Pflugers Arch. 444, 491-498.

Markram, H., Lubke, J., Frotscher, M., Roth, A., and Sakmann, B. (1997). Physiology and anatomy of synaptic connections between thick tufted pyramidal in the developing rat neocortex. J. Physiol. 500, 409-440.

Mason, A., Nicoll, A., and Stratford, K. (1991).Synaptic transmission between 
individual pyramidal neurons of the rat visual cortex in vitro. J. Neurosci. $11,72-84$.

Matsuzaki, M., Ellis-Davies, G. C., and Kasai, H. (2008). Three-dimensional mapping of unitary synaptic connections by two-photon macro photolysis of caged glutamate. J. Neurophysiol. 99, 1535-1544.

Mehring, C., Hehl, U., Kubo, M., Diesmann, M., and Aertsen, A. (2003). Activity dynamics and propagation of synchronous spiking in locally connected random networks. Biol. Cybern. 88, 395-408.

Mitchison, G., and Crick, F. (1982). Long axons within the striate cortex: their distribution, orientation, and patterns of connection. Proc. Natl. Acad. Sci. U.S.A. 79, 3661-3665.

Nawrot, M. P., Boucsein, C., Rodriguez, M.V., Riehle, A., Aertsen, A., and Rotter, S. (2008). Measurement of variability dynamics in cortical spike trains. J. Neurosci. Methods 169, 374-390.

Nikolenko, V., Poskanzer, K. E., and Yuste, R. (2007). Two-photon photostimulation and imaging of neural circuits. Nat. Methods 4, 943-950.

Nowak, L. G., Sanchez-Vives, M. V., and McCormick, D. A. (1997). Influence of low and high frequency inputs on spike timing in visual cortical neurons. Cereb. Cortex 7, 487-501.

Roy, S. A., and Alloway, K. D. (2001). Coincidence detection or temporal integration? What the neurons in somatosensory cortex are doing. J. Neurosci. 21, 2462-2473.

Schubert, D., Kotter, R., Zilles, K., Luhmann, H. J., and Staiger, J. F. (2003). Cell type-specific circuits of cortical layer IV spiny neurons. J. Neurosci. 23, 2961-2970.

Shadlen, M. N., and Newsome, W. T. (1994). Noise, neural codes and cortical organization. Curr. Opin. Neurobiol. 4, 569-579.

Shadlen, M. N., and Newsome, W. T. (1998). The variable discharge of cortical neurons: implications for connectivity, computation, and information coding. J. Neurosci. 18, 3870-3896.

Shoham, S., O'Connor, D. H., Sarkisov, D. V., and Wang, S. S. (2005). Rapid neurotransmitter uncaging in spatially defined patterns. Nat. Methods 2, 837-843.

Silberberg, G., Bethge, M., Markram, H., Pawelzik, K., and Tsodyks, M. (2004). Dynamics of population rate codes in ensembles of neocortical neurons. J. Neurophysiol. 91, 704-709.

Silver, R. A., Lubke, J., Sakmann, B., and Feldmeyer,D. (2003).High-probability uniquantal transmission at excitatory synapses in barrel cortex. Science 302, 1981-1984.

Song, S., Sjostrom, P. J., Reigl, M., Nelson, S., and Chklovskii, D. B. (2005). Highly nonrandom features of synaptic connectivity in local cortical circuits. PLoS Biol. 3, e68.

Staiger, J. F., Flagmeyer, I., Schubert, D., Zilles, K., Kotter, R., and Luhmann, H. J.
(2004). Functional diversity of layer IV spiny neurons in rat somatosensory cortex: quantitative morphology of electrophysiologically characterized and biocytin labeled cells. Cereb. Cortex 14, 690-701.

Tamas, G., Szabadics, J., and Somogyi, P. (2002). Cell type- and subcellular position-dependent summation of unitary postsynaptic potentials in neocortical neurons. J. Neurosci. 22, 740-747.

Thomson, A. M., and Bannister, A. P. (2003). Interlaminar connections in the neocortex. Cereb. Cortex 13, 5-14. von der Malsburg, C. (1986). Am I thinking assemblies? In Brain Theory, G. Palm and A. Aertsen, eds (Berlin, Springer), pp. 161-176.

Warren, M. A., and Bedi, K. S. (1982) Synapse-to-neuron ratios in the visual cortex of adult rats undernourished from about birth until 100 days of age. J. Comp. Neurol. 210, 59-64.

Waters, J., and Helmchen, F. (2006) Background synaptic activity is sparse in neocortex. J. Neurosci. 26, 8267-8277.

Weiler, N., Wood, L., Yu, J., Solla, S. A., and Shepherd, G. M. (2008). Top-down laminar organization of the excitatory network in motor cortex. Nat. Neurosci. 11, 360-366.

Weliky, M., and Katz, L. C. (1994). Functional mapping of horizontal connections in developing ferret visual cortex: experiments and modeling. J. Neurosci. 14, 7291-7305.
Williams, S. R., and Stuart, G. J. (2002). Dependence of EPSP efficacy on synapse location in neocortical pyramidal neurons. Science 295, 1907-1910.

Williams, S. R., and Stuart, G. J. (2003). Role of dendritic synapse location in the control of action potential output. Trends Neurosci. 26, 147-154.

Zador,A. (1998). Impact of synaptic unreliability on the information transmitted by spiking neurons. J. Neurophysiol. 79, 1219-1229.

Conflict of Interest Statement: The authors declare that the research was conducted in the absence of any commercial or financial relationships that could be construed as a potential conflict of interest.

Received: 15 September 2008; paper pending published: 28 October 2008; accepted: 27 January 2009; published: 10 February 2009.

Citation: Nawrot MP, Schnepel P, Aertsen $A$ and Boucsein C (2009) Precisely timed signal transmission in neocortical networks with reliable intermediate-range projections. Front. Neural Circuits (2009) 3:1. doi: 10.3389/neuro.04.001.2009 Copyright (c) 2009 Nawrot, Schnepel, Aertsen and Boucsein. This is an openaccess article subject to an exclusive license agreement between the authors and the Frontiers Research Foundation, which permits unrestricted use, distribution, and reproduction in any medium, provided the original authors and source are credited. 